\title{
Assessment Method of Wind Farm Harmonic Emission Value Based on Improved Complex Linear Regression Model
}

\author{
Tiantian Chen ${ }^{1,}$, Peng Zhang ${ }^{1}$, Ling Pan ${ }^{1}$, Peng Tan $^{2}$ and Honggen Yang ${ }^{2}$ \\ ${ }^{1}$ State Grid Shanghai Municipal Electric Power Company, Shanghai, 200437, China \\ ${ }^{2}$ School of Electrical and Information, Sichuan University, Chengdu, 610065, China
}

\begin{abstract}
Wind turbine filter and reactive power compensation devices lead to the harmonic impedance of wind farm is not much larger than that of the utility, so the influence by the wind farm harmonic impedance can not be neglected while assessing the harmonic emission value of wind farm A method based on improved complex linear regression is proposed in this paper for assessing the harmonic emission value of wind farm. The linear regression model is established by using the harmonic current at PCC point as the explanatory variable and the harmonic voltage of the wind farm as the explanatory variable. The utility harmonic impedance is calculated by complex least squares method. For various of the topology of wind farm feeder network, an equivalent method of feeder network is proposed to calculate the wind farm harmonic impedance. Errors are analyzed by using the error marginal effect of the dispersion parameter. Simulation and measured data verify the effectiveness of the proposed method
\end{abstract}

\section{Introduction}

LCL type grid-connected inverter of wind turbine will produce harmonics, witch lead to power quality and stability problems [1].The harmonics of the point of common coupling of wind farm is the result of the internal harmonic source of wind farm and the harmonic source of external power network. Therefore, it is necessary to reasonably classify the harmonic pollution responsibility of PCC point, the premise is to reasonably evaluate the harmonic emission level on both sides of PCC point [2].

A large amount of research on harmonic emission level evaluation methods was carried out. The customer and the utility reference impedance method was proposed in [3]. However, the operating conditions of generator of utility and the load of customer are changing. So, it is hard to calculate the reference impedance accurately according to the utility and customer parameters. Therefore, the non-invasive harmonic emission level assessment method based on the measurement data was proposed, including fluctuation method [4], linear regression method [5], independent random vector method [6], maximum likelihood estimation method [7] and independent component analysis method [8].The complex linear least square method proposed in [9] is the real solution in the complex domain calculation of least squares regression model, which maintains the strict linear relationship of the regression model and improves the weakness of least squares solution of regression model in the real domain. However, the accuracy of regression results are affected by the collinearity between the PCC harmonic current and utility harmonics. All the methods above assume that the customer harmonic impedance is much larger than the utility harmonic impedance, so that the customer harmonic impedance is neglected when calculating the harmonic emission level of customer. For typical industrial nonlinear customers (Industrial rectifier loads, arc furnaces, etc.), the above hypothesis holds. However, the wind turbine inverter is equipped with LCL-filter, and the collector point is equipped with reactive power compensation devices, which may cause the harmonic impedance of the wind farm is not much larger than that of the utility, so the influence by the wind farm harmonic impedance can not be neglected.

The harmonic impedance of the wind farm is mainly determined by the wind turbine LCL-filter, transformer, feeder line and reactive compensation capacitor, all of which have definite harmonic impedance model. The wind turbine of same type and capacity is equipped with LCL filters whose parameters are basically consistent. Only the length of line between the wind turbine and collecting point is dispersive, so the structure of the feeder network can be simplified and the harmonic impedance of the wind farm side can be calculated.

In this paper, an improved linear regression model is proposed by using the PCC point harmonic current as the explanatory variable and the harmonic voltage of the wind farm as the explanatory variable. The utility harmonic impedance is calculated by using the complex least square method. Aiming at the variability of topology structure of wind farm feeder network, an equivalent method of collector network is proposed to calculate the wind farm harmonic impedance. The error

\footnotetext{
* Corresponding author: author@e-mail.org
} 
margin effect of the dispersion parameter is used to analyze the error. Simulation and field data verify the effectiveness of the proposed method.

\section{Theory of improved complex linear regression model}

In this paper, Norton equivalent circuit is commonly used to perform harmonic analysis as shown in Fig.1,where $I_{U}$ and $Z_{U}$ are the utility-side equivalent harmonic current and impedance, $I_{C}$ and $Z_{C}$ are the wind farm-side equivalent harmonic current and impedance, and $U_{P C C}$ and $I_{P C C}$ are the harmonic voltage and current measured at the PCC.



Figure 1 Norton equivalent circuit of utility and wind farm

Referring to Fig. 1, the $I_{P C C}$ can be expressed as

$$
I_{P C C}=\frac{Z_{C}}{Z_{U}+Z_{C}} I_{C}-\frac{Z_{U}}{Z_{U}+Z_{C}} I_{U}
$$

According to Thevenin and Norton transformation, $I_{P C C}$ can be expressed as (2), where $V_{C}$ and $V_{U}$ are the equivalent utility-side and wind farm-side harmonic voltage.

$$
I_{P C C}=\frac{V_{C}}{Z_{U}+Z_{C}}-\frac{V_{U}}{Z_{U}+Z_{C}}
$$

According to Kirchhoff's current law, the wind farmside equivalent voltage $V_{C}$ can be calculated as

$$
V_{C}=U_{P C C}+Z_{C} I_{P C C}
$$

The wind farm-side equivalent harmonic impedance can be calculated by parameters of wind farm.

Due to the error of the measured data, the following linear relationship exists between $I_{P C C}$ and $V_{C}$ can be expressed as (4), where $\varepsilon$ is the measurement error.

$$
I_{P C C}=\frac{1}{Z_{U}+Z_{C}} V_{C}+\frac{-V_{U}}{Z_{U}+Z_{C}}+\varepsilon
$$

A linear regression equation between $I_{P C C}$ and $V_{C}$ can be established based on (4) as

$$
I_{P C C}=\beta_{1} V_{C}+\beta_{0}+\varepsilon
$$

where $\beta_{1}$ and $\beta_{0}$ are partial regression coefficient. $\beta_{1}=1 /\left(Z_{U}+Z_{C}\right), \beta_{0}=-V_{U} /\left(Z_{U}+Z_{C}\right)$.

During a measurement period, a $n$ group data of PCC point harmonic currents and wind farm side harmonic voltages can be expressed as

$$
\left\{\begin{array}{c}
I_{P C C}(1)=\beta_{1} V_{C}(1)+\beta_{0}+\varepsilon_{1} \\
I_{P C C}(2)=\beta_{1} V_{C}(2)+\beta_{0}+\varepsilon_{2} \\
\cdots \\
I_{P C C}(n)=\beta_{1} V_{C}(n)+\beta_{0}+\varepsilon_{n}
\end{array}\right.
$$

(6) can be represented as a matrix equation

$$
y=A x+\varepsilon
$$

Where $y=\left[I_{P C C}(1) I_{P C C}(2) \ldots I_{P C C}(n)\right]^{T}$,

$$
A=\left[\begin{array}{cc}
1 & \dot{I}_{C}(1) \\
1 & \dot{I}_{C}(2) \\
\vdots & \vdots \\
1 & \dot{I}_{C}(n)
\end{array}\right], x=\left[\begin{array}{c}
\beta_{0} \\
\beta_{1}
\end{array}\right], \varepsilon=\left[\begin{array}{c}
\varepsilon_{1} \\
\varepsilon_{2} \\
\vdots \\
\varepsilon_{n}
\end{array}\right] .
$$

In order to minimize the sum of squares of $\varepsilon$, the complex least squares method in [9] is used by

$$
x=\left(\bar{A}^{T} A\right)^{-1} \bar{A}^{T} y
$$

where "' $^{-}$" stands for conjugate vector.

Compared with the method in [9], there is no correlation between the harmonic current of the wind farm-side and the utility-side harmonic voltage. For the regression results of the equation (7) are not interfered with by the collinearity factor, the regression result is more accurate.

Based on the regression result of $\beta_{1}$, the utility harmonic impedance can be calculated by

$$
Z_{U}=\frac{1}{\beta_{1}}-Z_{C}
$$

By the equivalent circuit, the harmonic voltage of PCC point can be expressed as

$$
U_{P C C}=\frac{Z_{C} Z_{U}}{Z_{U}+Z_{C}} I_{C}+\frac{Z_{C} Z_{U}}{Z_{U}+Z_{C}} I_{U}
$$

According to the principle of superposition, the harmonic voltage emission value of the wind farm side is

$$
U_{P C C-\mathrm{C}}=\frac{Z_{U} Z_{\mathrm{C}}}{Z_{U}+Z_{\mathrm{C}}}\left(\frac{U_{P C C}}{Z_{\mathrm{C}}}+I_{P C C}\right)
$$

And the harmonic voltage emission value of the utility side is

$$
\dot{U}_{P C C-\mathrm{S}}=\dot{U}_{P C C}-\dot{U}_{P C C-\mathrm{C}}
$$

\section{Calculation of the wind farm side harmonic impedance}

\subsection{Harmonic model of wind farm}

Taking an actual wind farm as an example, the wind farm consists of two parts, one part is the feeder network, which is composed of the wind turbine, transformer and feeder line, the other part is the reactive power compensation device, as shown in Fig.2

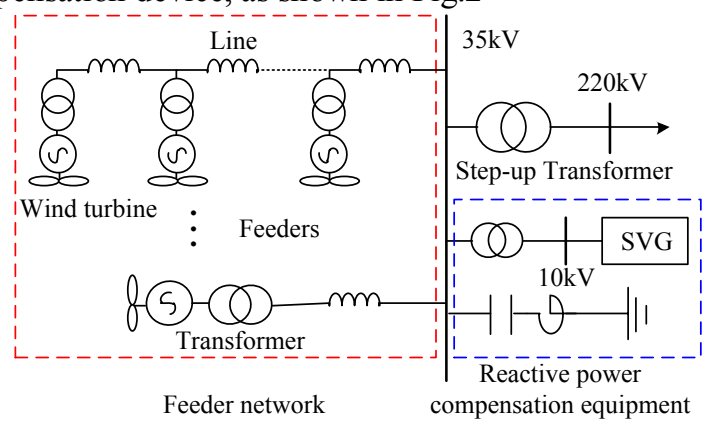

Figure 2 Topology of wind farm power station

\subsection{Harmonic Impedance Model of PMSG}


The permanent magnet synchronous generator (PMSG) consists of wind turbine, permanent magnet synchronous generator, generator side converter, grid side converter and LCL filter. Since the generator side converter and the grid side converter are separated by DC capacitor, the harmonic characteristics of wind turbine are determined only by the harmonic characteristics of the LCL inverter on the grid side.

The harmonic voltage of grid side inverter consists of two parts, one part $U_{i n v}$, is generated by the control system of inverter the other part $U_{p w m}$, is generated by the dead time and modulation process of inverter. Using the inverter harmonic voltage source and LCL filter to represent the wind turbine harmonic impedance model [10] as shown in Fig.4.

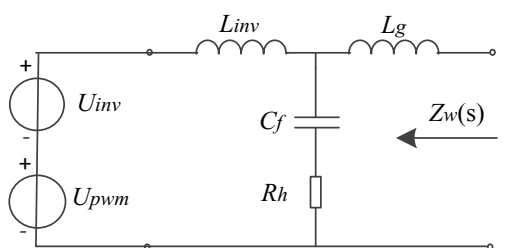

Figure 4 Harmonic model of PMSG-based wind turbine

Where, $L_{i n v}$ and $L_{g}$ are the inverter side and grid side inductance, $C_{f}$ is the filter capacitor, $R_{h}$ is the damping resistance.

The harmonic impedance of wind turbine can be obtained by the LCL filter parameter as follows:

$$
Z_{W}(w)=j w L_{g}+\frac{-w^{2} R_{h} C_{f} L_{i n v}+j w L_{i n v}}{-w^{2} L_{i n v} C_{f}+j w R_{h} C_{f}+1}
$$

Where, $w$ stands for the frequency.

\subsection{Harmonic Impedance Model of SVG}

The SVG outputs the reactive current through the inverter, in order to suppress the harmonics produced by SVG inverters, L filters are usually connected between the grid and the inverter. In practical engineering, the SVG connection transformer is commonly used as the filter inductance. Accordingly, the harmonic impedance model of SVG is a series harmonic model consisting of the equivalent harmonic voltage source of the inverter and the short-circuit impedance of the transformer connected with the SVG. It can be de defined as follows:

$$
Z_{S V G}(w)=j w L_{T}+R_{T}
$$

Where $L_{T}$ and $R_{T}$ are the equivalent inductance and resistance of transformer.

\subsection{Harmonic Impedance of wind farm side}

The impedance of the feeder network can be accurately calculated according to the parameters of the LCL filter, the line impedance and the detailed topology of the feeder network. The harmonic impedance of reactive power compensation device can be calculated, according to the detail parameters. When the measurement point is located on the collector point, the current measured is the total current of the feeder network and the reactive compensation device, the harmonic impedance of the wind farm-side is the parallel total impedance of the feeder network and reactive power compensation devices.

\section{Approximate calculation of the wind farm-side harmonic impedance}

In the actual project, it is difficult to obtain the detailed topology of the feeder network, which needs to approximate the harmonic impedance of the feeder network.

\subsection{Aggregation equivalence method of feeder network}

The topology of the feeder network is changeable, but the parameters of the LCL filter are consistent, only the length of line between the wind turbine and collecting point is dispersive. The equivalent circuit of a single feeder is shown in Fig 5.

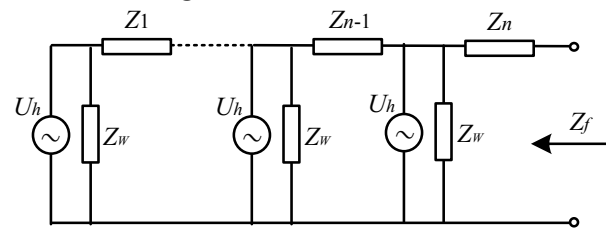

Figure 5 Equivalent circuit diagram of single feeder

Where $n$ is the number of the wind turbines, $Z_{W}$ is the impedance of a single wind turbine, $U_{h}$ is the harmonic voltage from a single wind turbine. $Z_{n}(n=1,2,3 \ldots n)$ is the impedance of the feeder line between adjacent wind turbines, whose sum is the impedance of the feeder line $Z_{L} . Z_{f}$ is the impedance of a single feeder.

The impedance of the feeder line is mixed by the wind turbine impedance and the line impedance. Considering that $Z_{W}$ is much larger than $Z_{L}$, the impedance of single feeder can be simplified as follows:

$$
Z_{f}=\left(Z_{W}+Z_{e q}\right) / n
$$

Where, $Z_{e q}$ is the equivalent line impedance of a single feeder, it can be obtained by

$$
Z_{e q} \approx \sum_{i=1}^{n} i Z_{i}=n Z_{L}-\sum_{i=1}^{n}(n-i) Z_{i}
$$

By (15), when $Z_{i}=0(i=1,2 \ldots n-1), Z_{n}=Z_{L}$, that is, the wind turbines are arranged at the end of the feeder, $Z_{e q}$ obtains the maximum value as $n Z_{L}$. when $Z_{i}=0(i=$ $2,3 \ldots n), Z_{1}=Z_{L}$, that is, only one wind turbine is arranged at the end of the feeeder, and the other wind turbines are arranged at the head of the feeder, $Z_{e q}$ obtains the minimum value as $Z_{L}$. The boundary value of $Z_{e q}$ can be expressed as follows:

$$
Z_{L} \leq Z_{e q} \leq n Z_{L}
$$

In order to minimize the impedance error of the feeder network between the approximation and the true values, the impedance approximation of feeder network $Z_{F J S}$ is defined as the mean value of the maximum and minimum by

$$
Z_{F J S}=\left(Z_{W}+\frac{Z_{L \min }+n_{\max } Z_{L \max }}{2}\right) / N
$$


Where, $Z_{L \min }$ and $Z_{L \max }$ are the impedance of the shortest and the longest feeder lines. $n_{\max }$ is the maximum number of wind turbines connected to a single feeder.

\subsection{Approximate Error of Harmonic Impedance in feeder Network}

Take an actual wind farm as an example,there is 5 feeders in the wind farm, each of which is equipped with 10 wind turbines. The length of feeder line is $4.7 \mathrm{~km}$, $8.0 \mathrm{~km}, \quad 11.2 \mathrm{~km}, \quad 13.5 \mathrm{~km}, 15.5 \mathrm{~km}, \quad$ respectively. Parameters of wind turbine and LCL filters is shown in Table1. The short circuit inductance of step-up transformer is used as the grid side inductance of LCL filters, whose value is $0.041 \mathrm{mH}$.

Table 1. Parameters of wind turbine and filter

\begin{tabular}{lc}
\hline \multicolumn{1}{c}{ Parameters } & Value \\
\hline Rated Power P/MW & 2.0 \\
Rated Voltage $U_{g} / \mathrm{kV}$ & 0.69 \\
Rated Capacity $S_{T} / \mathrm{MVA}$ & 2.2 \\
Rated Voltage of transformer $U_{T} / \mathrm{kV}$ & $0.69 / 37$ \\
Short-circuit impedance of transformer $\%$ & $6 \%$ \\
Inverter side inductance $L_{i n} / \mathrm{mH}$ & 0.18 \\
Grid side inductance $L_{g} / \mathrm{mH}$ & 0.04 \\
Filter capacitor $C_{f} / \mathrm{uF}$ & 700 \\
Filter resistor $R_{h} / \Omega$ & 0.1 \\
\hline
\end{tabular}

Consider the extreme conditions that may exist in the topology of feeder network: the wind turbines adopts an end or head arrangement, and the length of the feeder 1 is the shortest or the longest. The error of primary harmonic approximate impedance of the feeder network is shown in table2.

Table 2. Errors of harmonic impedance approximation of feeder network

\begin{tabular}{|c|c|c|c|c|c|}
\hline \multicolumn{6}{|c|}{$\begin{array}{c}\text { Errors of Harmonic Impedance Approximation of Feeder } \\
\text { Network (\%) }\end{array}$} \\
\hline \multicolumn{2}{|c|}{ Harmonic order } & $5 t h$ & $7 t h$ & 11th & $13 t h$ \\
\hline \multirow{2}{*}{$\begin{array}{c}\text { End } \\
\text { arrangement }\end{array}$} & Longest line & -0.5 & -0.35 & 0.5 & 1.1 \\
\hline & Shortest line & 10.0 & 6.2 & -6.9 & -16.1 \\
\hline \multirow{2}{*}{$\begin{array}{c}\text { Head } \\
\text { rangement }\end{array}$} & Longest line & -5.1 & -.32 & 3.8 & 10.2 \\
\hline & Shortest line & -4.1 & -2.6 & 3.1 & 7.8 \\
\hline \multicolumn{2}{|c|}{ Accurate value } & -1.1 & -0.7 & 0.7 & 2.0 \\
\hline
\end{tabular}

Taking the 5th harmonic as an example, in order to show the relationship between the feeder network impedance and the number of wind turbines, the harmonic admittance of the feeder network is shown in Fig.6.

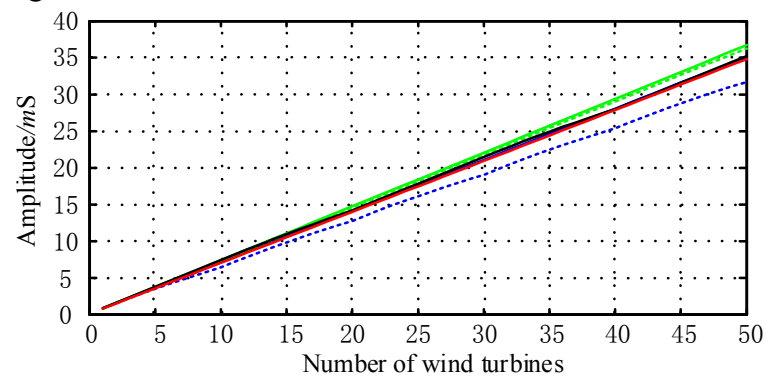

Figure 6 Amplitude of feeder network of 5th harmonic admittance

Where, the red curve represents the approximate value of admittance, and the black curve represents the exact value. The green and blue curves represent the end and head arrangement of wind turbines, respectively. The corresponding solid lines and dashed lines represent the conditions of shortest and longest lines.

Obviously, with the increase of the number of the wind turbine, the accurate harmonic admittance of the feeder network almost increases linearly, and the maximum error of the approximate harmonic impedance value is only $-16.1 \%$.

\subsection{Approximation of wind farm-side harmonic impedance} table3.

Other parameters of the wind farm are shown in

Table 3. Parameters of wind farm

\begin{tabular}{|c|c|}
\hline Parameters & Parameter Description \\
\hline Short-circuit & $35 \mathrm{kV}: 766 \mathrm{MVA}$ \\
\hline Capacity & $220 \mathrm{kV}: 11090 \mathrm{MVA}$ \\
\hline $\begin{array}{l}\text { Step-up } \\
\text { Transformer }\end{array}$ & $\begin{array}{l}\text { Rated Voltage } 230 \pm 8 \times 1.25 \% / 37 \mathrm{kV} \text {, Rated } \\
\text { Capacity } 100 \mathrm{MVA} \text {, Connection Sets: Ynyn0, } \\
\text { Short-circuit Impedance of Transformer: } 6 \%\end{array}$ \\
\hline Fixed & Rated Capacity 12Mvar, Capacitor Series \\
\hline Capacitor & Reactance Rate: $6 \%$, Quality Factor: 100. \\
\hline SVG & Rated Capacity 9Mvar, Rated Voltage: $10 \mathrm{kV}$ \\
\hline $\begin{array}{l}\text { SVG } \\
\text { Transformer }\end{array}$ & $\begin{array}{l}\text { Rated Voltage } 37 \pm 2 \times 2.5 \% / 10 \mathrm{kV} \text {, Rated Capacity } \\
\text { 10MVA, Connection Sets: Ynyn0, Short-circuit } \\
\text { Impedance of Transformer: } 10 \%\end{array}$ \\
\hline
\end{tabular}

When the measurement point is located in the $35 \mathrm{k} V$ bus (collecting point), the minimum, maximum, accurate and approximate values of the primary harmonic impedances of wind farms side are shown in table 4

Table 4. Harmonic impedance boundary value of wind farm

\begin{tabular}{c|llll}
\hline \multicolumn{4}{c}{ Harmonic impedance boundary value of wind farm $(\Omega)$} \\
\hline $\begin{array}{c}\text { Harmonic } \\
\text { order }\end{array}$ & $\begin{array}{c}\text { Minimum } \\
\text { value }\end{array}$ & $\begin{array}{c}\text { maximum } \\
\text { value }\end{array}$ & $\begin{array}{c}\text { Accurate } \\
\text { value }\end{array}$ & $\begin{array}{c}\text { Approximate } \\
\text { value }\end{array}$ \\
\hline 5th & $7.8 \angle 88^{\circ}$ & $8.1 \angle 88^{\circ}$ & $7.9 \angle 88^{\circ}$ & $7.9 \angle 88^{\circ}$ \\
7 th & $20.1 \angle 86^{\circ}$ & $20.8 \angle 86^{\circ}$ & $20.3 \angle 86^{\circ}$ & $20.4 \angle 86^{\circ}$ \\
11th & $64.4 \angle 17^{\circ}$ & $79.6 \angle 19^{\circ}$ & $74.9 \angle 18^{\circ}$ & $74.2 \angle 18^{\circ}$ \\
13th & $26.3 \angle-14^{\circ}$ & $40.9 \angle-30^{\circ}$ & $35.7 \angle-25^{\circ}$ & $34.9 \angle-25^{\circ}$ \\
\hline
\end{tabular}

From table 4 , the maximum amplitude error of the 5 th, 7th, 11th, 13th harmonic impedance approximation is $2.5 \%, 1.9 \%, 13.2 \%$ and $24.6 \%$, respectively.

The utility harmonic impedances can be roughly calculated by the short-circuit capacity of measuring point, which are $9,13,20,23 \Omega$ for 5 th, 7 th, 11th, 13th harmonic respectively. So, the amplitude of wind farm harmonic impedance is not much larger than the utility side.

\section{Harmonic impedance boundary value of wind farm with parameter error}

There is an error between the actual parameters and the rated parameters of the component, resulting in a change in the impedance of the wind turbine within a certain range. There is a error in the capacity of filter inductance and filter capacitor, which is generally 
believed to $5 \%$ as maximum [11]. And there is a error in short-circuit impedance of transformer, which is generally believed to $10 \%$ as maximum.

The inductance of the filter, the capacity of the capacitor, and the short-circuit impedance of transformer may increase or decrease at the same time. Based on analysis and comparison, the $5^{\text {th }}, 7^{\text {th }}$ harmonic impedance increase and the $11^{\text {th }}, 13^{\text {th }}$ harmonic impedance decrease, when $L_{i n v}, C_{f}$ and $L_{g}$ increase, vice versa.

When the filter parameter error is the maximum value, the boundary value of single wind turbine is shown in Fig7.

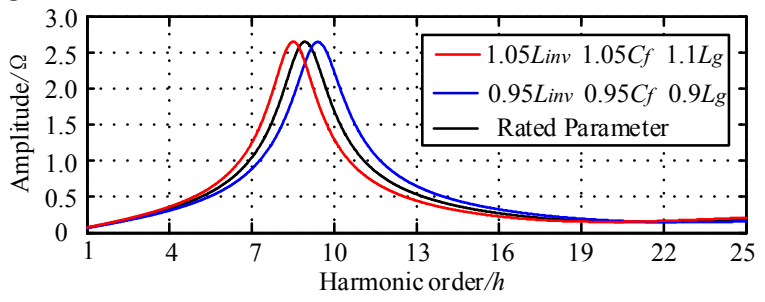

Figure 7 Amplitude of wind turbine harmonic impedance

In order to avoid resonance between filter and power grid, the tuning point is designed on the frequency of non integer harmonic according to the principle of partial resonance.

As shown in Fig8, the actual resonance point of the filter is designed between $430 \mathrm{~Hz}-471 \mathrm{~Hz}$ with parameter errors of LCL filter, witch is from the primary integer harmonics.

Therefore, the error of filter parameters will not lead to changes in impedance properties of wind turbine. So, there is no interference between the inductive and capacitive impedances of wind turbines.

The boundary value of the impedance of the feeder network can be obtained by the boundary value of the impedance of each wind turbine.

Considering the parameter error of component and the approximation error of the feeder network, the boundary error of the harmonic impedance of the feeder network and the corresponding boundary value of harmonic impedance of the wind farm can be calculated as shown in table 5 and table 6 , respectively.

Table 5. Harmonic impedance boundary error of feeder network

\begin{tabular}{l|cccc}
\hline \multicolumn{5}{c}{ Harmonic impedance boundary error of feeder network(\%) } \\
\hline Harmonic order & 5th & 7 th & 11th & 13th \\
\hline Minimum value & -13.6 & -17.9 & -28 & -32 \\
maximum value & 19.2 & 25.1 & 34.2 & 33.9 \\
\hline
\end{tabular}

Table 6. Harmonic impedance boundary value of wind farm with parameter error

\begin{tabular}{c|cccc}
\hline \multicolumn{5}{c}{ Harmonic impedance boundary value of wind farm $(\Omega)$} \\
\hline $\begin{array}{c}\text { Harmonic } \\
\text { order }\end{array}$ & $\begin{array}{c}\text { Minimum } \\
\text { value }\end{array}$ & $\begin{array}{c}\text { maximum } \\
\text { value }\end{array}$ & $\begin{array}{c}\text { Accurate } \\
\text { value }\end{array}$ & $\begin{array}{c}\text { Approximate } \\
\text { value }\end{array}$ \\
\hline 5th & $7.6 \angle 88^{\circ}$ & $8.3 \angle 88^{\circ}$ & $7.9 \angle 88^{\circ}$ & $7.9 \angle 88^{\circ}$ \\
7th & $19.0 \angle 86^{\circ}$ & $21.8 \angle 85^{\circ}$ & $20.3 \angle 86^{\circ}$ & $20.4 \angle 86^{\circ}$ \\
11th & $52.7 \angle 3^{\circ}$ & $78.9 \angle 37^{\circ}$ & $74.9 \angle 18^{\circ}$ & $74.2 \angle 18^{\circ}$ \\
13th & $20.2 \angle-10^{\circ}$ & $52.9 \angle-24^{\circ}$ & $35.7 \angle-25^{\circ}$ & $34.9 \angle-25^{\circ}$ \\
\hline
\end{tabular}

From table 6 , the maximum error of approximate value of wind farm impedance is $5 \%, 7 \%, 30 \%$ and $52 \%$ for 5 th, 7th, 11th, 13th harmonic respectively.

\section{Simulation and field data analysis}

\subsection{Simulation analysis}

A computer simulation study was performed by Matlab7.0, which was based on the Norton equivalent circuit shown in Fig.1, including 1440 sample points at PCC.

The parameter was set as follows (the percentage of disturbance indicates the maximum of uniform disturbance comparing to initial value):

1) The amplitude of $I_{C}$ is $150 \mathrm{~A}$, and that of $I_{U}$ is 0.5 times smaller. Phase angles of $I_{C}$ and $I_{U}$ are respectively and $30^{\circ}$ and $50^{\circ} .10 \%$ sine fluctuation and $10 \%$ random disturbance are added to the phase angles of $I_{C .} .5 \%$ sine fluctuation and $20 \%$ random disturbance are added to the amplitude of $I_{C} .10 \%$ sine fluctuation and $10 \%$ random disturbance are added to the phase angles of $I_{U} .5 \%$ sine fluctuation and $10 \%$ random disturbance are added to the amplitude of $I_{U}$

2) The amplitude of $Z_{U}$ is set as the rough values of utility harmonic impedance in the foregoing paragraph. Phase angles of $Z_{U}$ is $70^{\circ} .10 \%$ sine fluctuation and $20 \%$ sine fluctuation are added to amplitude and angles of $Z_{U}$ respectively. $Z_{C}$ is set as the values in tableV and tableVI.

With 100 sample points for each subinterval, sliding analysis is performed. The results errors of method in [9] (method a) and method proposed in this paper (method b) was compared.With the approximate error of feeder network is taken into account, the errors of utility impedance and harmonic voltage emission value of wind farm are shown in table 7 and table 8 .

Table 7. Errors of utility harmonic impedance amplitude

\begin{tabular}{|c|c|c|c|c|c|c|}
\hline \multicolumn{7}{|c|}{ Root mean square value of the relative error $(\%)$} \\
\hline$Z_{C}$ & \multicolumn{2}{|c|}{ Minimum value } & \multicolumn{2}{|c|}{ maximum value } & \multicolumn{2}{|c|}{ Accurate value } \\
\hline Method & $a$ & $b$ & $a$ & $b$ & $a$ & $b$ \\
\hline 5 th & 10.2 & 5.1 & 10.5 & 5.3 & 9.0 & 4.1 \\
\hline $7 t h$ & 8.9 & 5.4 & 9.2 & 5.3 & 8.7 & 3.5 \\
\hline 11th & 7.8 & 4.6 & 5.6 & 3.6 & 7.2 & 3.2 \\
\hline 13th & 8.6 & 4.4 & 9.0 & 4.5 & 7.5 & 3.8 \\
\hline
\end{tabular}

Table 8. Errors of harmonic voltage emission value of wind farm

\begin{tabular}{c|ll|ll|ll}
\hline \multicolumn{6}{c|}{ Root mean square value of the relative error (\%) } \\
\hline $\boldsymbol{Z}_{\boldsymbol{C}}$ & \multicolumn{2}{|c|}{ Minimum value } & \multicolumn{2}{|c|}{ maximum value } & \multicolumn{2}{|c}{ Accurate value } \\
\hline Method & \multicolumn{1}{c}{$\boldsymbol{a}$} & $\boldsymbol{b}$ & $\boldsymbol{a}$ & $\boldsymbol{b}$ & $\boldsymbol{a}$ & $\boldsymbol{b}$ \\
\hline 5th & 52.2 & 3.1 & 49.8 & 3.5 & 50.9 & 3.4 \\
$\mathbf{7} \boldsymbol{t h}$ & 30.8 & 3.2 & 28.1 & 2.8 & 27.8 & 3.0 \\
11th & 5.6 & 3.6 & 5.2 & 3.9 & 4.8 & 3.2 \\
13th & 28.2 & 10.8 & 29.1 & 5.4 & 30.4 & 4.6 \\
\hline
\end{tabular}

With the approximate error of feeder network and the parameter errors of LCL filters are taken into account, the errors of utility impedance and harmonic voltage emission value of wind farm are shown in table 9 and table 10.

Table 9. Errors of utility harmonic impedance amplitude

\begin{tabular}{c|cc|cc|cc}
\hline \multicolumn{6}{c}{ Root mean square value of the relative error $(\%)$} \\
\hline $\boldsymbol{Z}_{\boldsymbol{C}}$ & \multicolumn{2}{|c|}{ Minimum value } & \multicolumn{2}{c}{ maximum value } & \multicolumn{2}{c}{ Accurate value } \\
\hline Method & $\boldsymbol{a}$ & $\boldsymbol{b}$ & $\boldsymbol{a}$ & $\boldsymbol{b}$ & $\boldsymbol{a}$ & $\boldsymbol{b}$ \\
\hline 5th & 8.9 & 4.8 & 11.3 & 5.9 & 9.0 & 4.1 \\
$\mathbf{7 t h}$ & 8.0 & 5.3 & 8.9 & 6.1 & 8.7 & 3.5 \\
11th & 7.5 & 4.3 & 10.3 & 6.0 & 7.2 & 3.2 \\
13th & 8.0 & 5.0 & 6.8 & 4.3 & 7.5 & 3.8 \\
\hline
\end{tabular}


Table 10. Errors of harmonic voltage emission value of wind farm

\begin{tabular}{|c|c|c|c|c|c|c|}
\hline \multicolumn{7}{|c|}{ Root mean square value of the relative error $(\%)$} \\
\hline \multirow{2}{*}{$\begin{array}{c}Z_{C} \\
\text { Method }\end{array}$} & \multicolumn{2}{|c|}{ Minimum value } & \multicolumn{2}{|c|}{ maximum value } & \multicolumn{2}{|c|}{ Accurate value } \\
\hline & $a$ & $b$ & $a$ & $b$ & $a$ & $b$ \\
\hline 5th & 52.1 & 3.6 & 48.2 & 3.5 & 50.5 & 3.0 \\
\hline $7 t h$ & 30.6 & 2.2 & 25.0 & 5.7 & 29.1 & 6.7 \\
\hline 11th & 5.3 & 3.2 & 7.2 & 5.8 & 4.6 & 3.3 \\
\hline 13th & 28.2 & 17.4 & 26.6 & 4.7 & 29.8 & 4.3 \\
\hline
\end{tabular}

It can be easily learned from table VII-X that method $\mathrm{b}$ has the smaller errors than method a, no matter the harmonic impedance of wind farm is the accurate value or the boundary value.

The actual impedance of the wind farm is within the range of the maximum and minimum boundary values. So, the method proposed in this paper has smaller errors of the utility harmonic impedance and the harmonic voltage emission value.

\subsection{Field data analysis}

To further verify effectiveness of the proposed method, field data are used to perform calculation. The measured data are drawn from the collecting point of the actual wind farm described above. The harmonic current of the feeder network and the harmonic voltage of PCC point is sampled. Harmonic data are acquired by using FFT algorithm to analyze sample data per minute. The 5 th harmonic current and voltage is shown in Fig8.

Method proposed in this paper and method in [9] are used to calculate the utility impedance and the harmonic voltage emission value of wind farm as shown in Fig9.

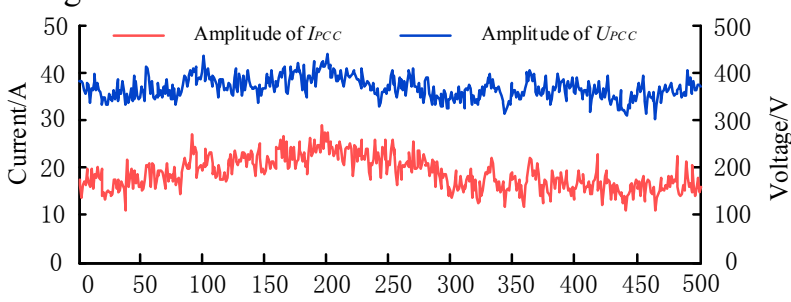

Figure 85 th harmonic voltage and current of PCC

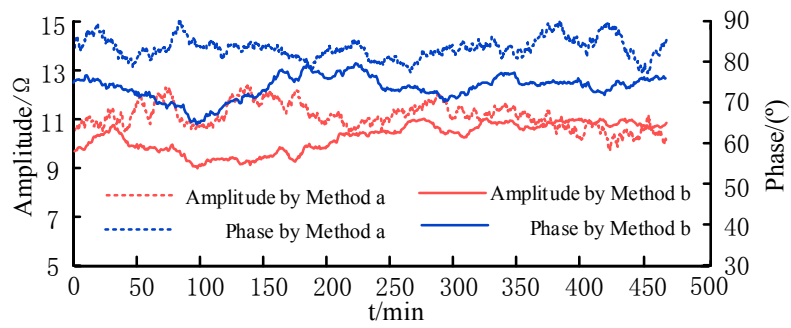

Figure 9 Estimates of the 5th utility harmonic impendance

The utility and wind farm side harmonic voltage emission value (95\% probability value) obtained by the method in [9] are respectively $231 \mathrm{~V}$ and $174 \mathrm{~V}$. However the utility and wind farm side harmonic voltage emission value (95\% probability value) obtained by the method proposed by this paper are respectively $308 \mathrm{~V}$ and $79 \mathrm{~V}$, which is quite different. Based on the analysis result of the simulations, the method proposed is more accurate.

\section{Conclusion}

In this paper, an assessment method of wind farm harmonic emission value based on improved complex linear regression model is proposed. Simulation and field data analysis verify the effectiveness and superiority of the proposed method.How to calculate the impedance of wind farm easily in engineering survey, through the component parameters acquired by the wind farm is the further research direction

\section{References}

1. Shuai Z, Liu D, Shen J, et al. Series and Parallel Resonance Problem of Wideband Frequency Harmonic and Its Elimination Strategy[J]. IEEE Transactions on Power Electronics, 2013, 29(4): 1941-1952.

2. Yang Honggeng, Wang Lei. Assessing harmonic emission levels from non-loads using Laguerre polynomials [J]. Proceedings of the CSEE, 2005, 25(7): 81-85.

3. $\mathrm{Xu} \mathrm{W}$, Liu Y. A method for determining customer and utility harmonic contributions at the point of common coupling [J]. IEEE Transactions on Power Delivery, 2000, 15(2):804-811.

4. Hui Jin, Yang Honggeng, Ye Maoqing. Assessing harmonic emission level based on the impedance gathering trend discrimination $[\mathrm{J}]$ Proceedings of the CSEE, 2011, 31(10): 73-80.

5. Zhang Wei, Yang Honggeng. A method for assessing harmonic emission level based on binary linear regression [J]. Proceedings of the CSEE, 2004, 4(6): 50-53.

6. Hui J, Yang H G, Lin S F, et al. Assessing utility harmonic impedance based on the covariance characteristic of random vectors [J]. IEEE Transactions on Power Delivery, 2010, 25(3): 17781786.

7. Hua Huichun, JiaXiufang, Cao Dongsheng, et al. A maximum likelihood method for harmonic impedance estimation [J]. Proceedings of the CSEE, 2014, 34(10): 1692-1699.

8. Zhao X, Yang H. A New Method to Calculate the Utility Harmonic Impedance Based on FastICA [J]. IEEE Transactions on Power Delivery, 2016, 31(1): 381-388.

9. Jia Xiufang, Hua Huichun, Cao Dongsheng, et al. Deterrmining harmonic contributions based on complex least squares method [J]. Proceedings of the CSEE, 2013, 33(4): 149-155.

10. Liu Z, Rong J, Zhao G, et al. Harmonic Assessment for Wind Parks Based on Sensitivity Analysis [J]. IEEE Transactions on Sustainable Energy, 2017, PP(99):1-1.]

11. Xu Mingjin, Ji Lin, Ge Xiaowei, et al. LCL-filter optimization design with consideration of inverterside current feedback control impacts [J]. Proceedings of the CSEE, 2016, 36(17): 4656-4664 